\title{
Enhanced Recovery after Cardiac Surgery: An Update on Clinical Implications
}

\section{Longqiu Yang, MD}

Department of Anesthesiology, Huangshi Central Hospital, Huangshigang, Huangshi Shi, Hubei Province, China

\section{Alan D. Kaye, MD, PhD}

Department of Anesthesiology, LSUHSC-New Orleans, New Orleans, Louisiana

\section{Archana G. Venakatesh, MD Michael S. Green, DO, MBA}

Department of Anesthesiology and Perioperative Medicine, Drexel University College of Medicine, Hahnemann University Hospital, Philadelphia, Pennsylvania

\section{Camellia D. Asgarian, MD}

Department of Anesthesiology, LSUHSC-New Orleans, New Orleans, Louisiana

\section{Markus M. Luedi, MD}

Department of Anesthesiology, Bern University Hospital Inselspital, University of Bern, Bern, Switzerland

\section{Henry Liu, MD}

Department of Anesthesiology and Perioperative Medicine, Drexel University College of Medicine, Hahnemann University Hospital, Philadelphia, Pennsylvania

\section{- Background}

Tremendous changes have occurred in enhanced recovery after surgery (ERAS) since Danish surgeon Dr Kehlet published his landmark article in 1997, emphasizing that major surgical procedures may lead to significant undesirable sequelae, including infection, pain, hemorrhage, cardiopulmonary and thromboembolic complications, ileus, postoperative nausea and vomiting, fatigue, and prolonged convalescence. ${ }^{1}$ In this regard, the surgical stress response and its subsequently increased demands on organ function is the critical factor influencing postoperative

\footnotetext{
Address Correspondence to: Henry Liu, mD, Department of Anesthesiology and Perioperative Medicine, Hahnemann University Hospital, Drexel University College of Medicine, 245 N. 15th Street, MS 310, Philadelphia, PA 19102. E-mail: henRyliupa@gmail.com
}

INTERNATIONAL ANESTHESIOLOGY CLINICS

Volume 55, Number 4, 148-162, DOI:10.1097/AIA.0000000000000168

Copyright (C) 2017 Wolters Kluwer Health, Inc. All rights reserved.

148 | www.anesthesiaclinics.com

Copyright (C) 2017 Wolters Kluwer Health, Inc. All rights reserved.

This paper can be cited using the date of access and the unique DOI number which can be found in the footnotes. 
morbidity and mortality. ${ }^{1}$ Significant physiological derailment is mediated by surgery and stress-induced endocrine/metabolic changes via activation of multiple biological cascades, including hormones, arachidonic acid metabolites, cytokines, complement, nitric oxide, and free oxygen radicals. ${ }^{2}$ ERAS adopts multidisciplinary approaches, multisystemic methods, and multimodal interventions to decrease postoperative morbidity and mortality, to remarkably improve recovery, and to reduce overall costs. ${ }^{2,3}$ Over the last 2 decades, ERAS programs have achieved accelerated recovery of systemic functions and improved clinical outcomes. ${ }^{4,5}$ However, for nonspecific reasons, cardiac surgery has lagged behind in establishing similar ERAS protocols. Some studies have attempted to establish an evidence-based ERAS protocol in cardiac surgery. ${ }^{6,7}$ The ERAS goals in cardiac surgery include decreased complications, shortened intensive care unit (ICU) and hospital stay, facilitation of functional recovery, and improved perioperative patient experience and satisfaction (Fig. 1).

\section{- Perioperative Stress Responses}

\section{Physiological Alterations}

The fight-or-flight responses activate the human autonomic nervous system perioperatively. As Figure 2 illustrates, perioperative stress can activate the autonomic nervous system and the hypothalamic-pituitaryadrenal gland axis. ${ }^{8}$ Perioperative stress and surgical injury can lead to an immunoinflammatory response. The initial response is predominantly an excessive activation of innate immune pathways manifested by a pronounced systemic inflammatory response and potential organ injury. Later, patients will have a persistent depression in adaptive immunity, greater suseptibility to nosocomial infections, ${ }^{9}$ and typically depression in $\mathrm{T}$-cell responses. Recent studies indicate that innate immune receptors detect tissue injury, such as Toll-like receptor

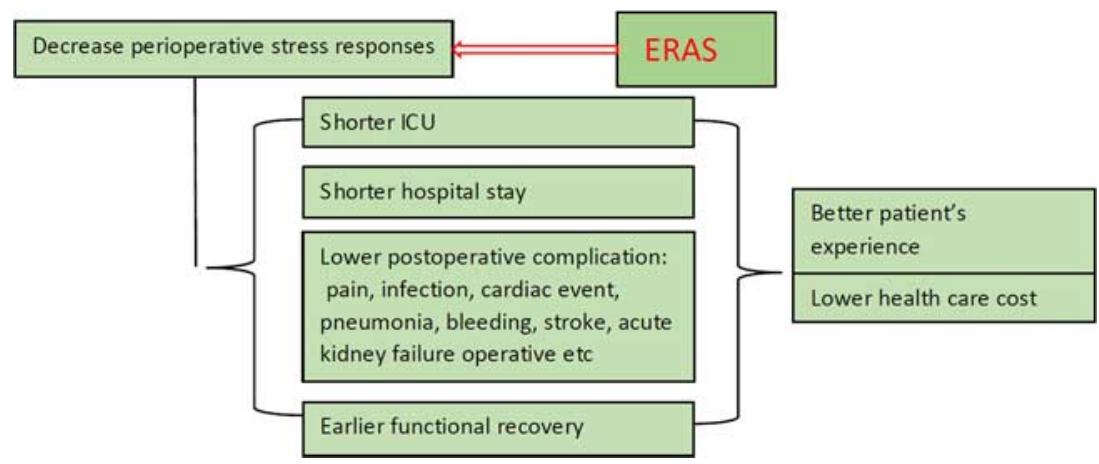

Figure 1. Overall goals for ERAS. ERAS indicates enhanced recovery after surgery; ICU, intensive care unit. $\frac{\text { full color }}{\mathrm{online}}$

www.anesthesiaclinics.com 


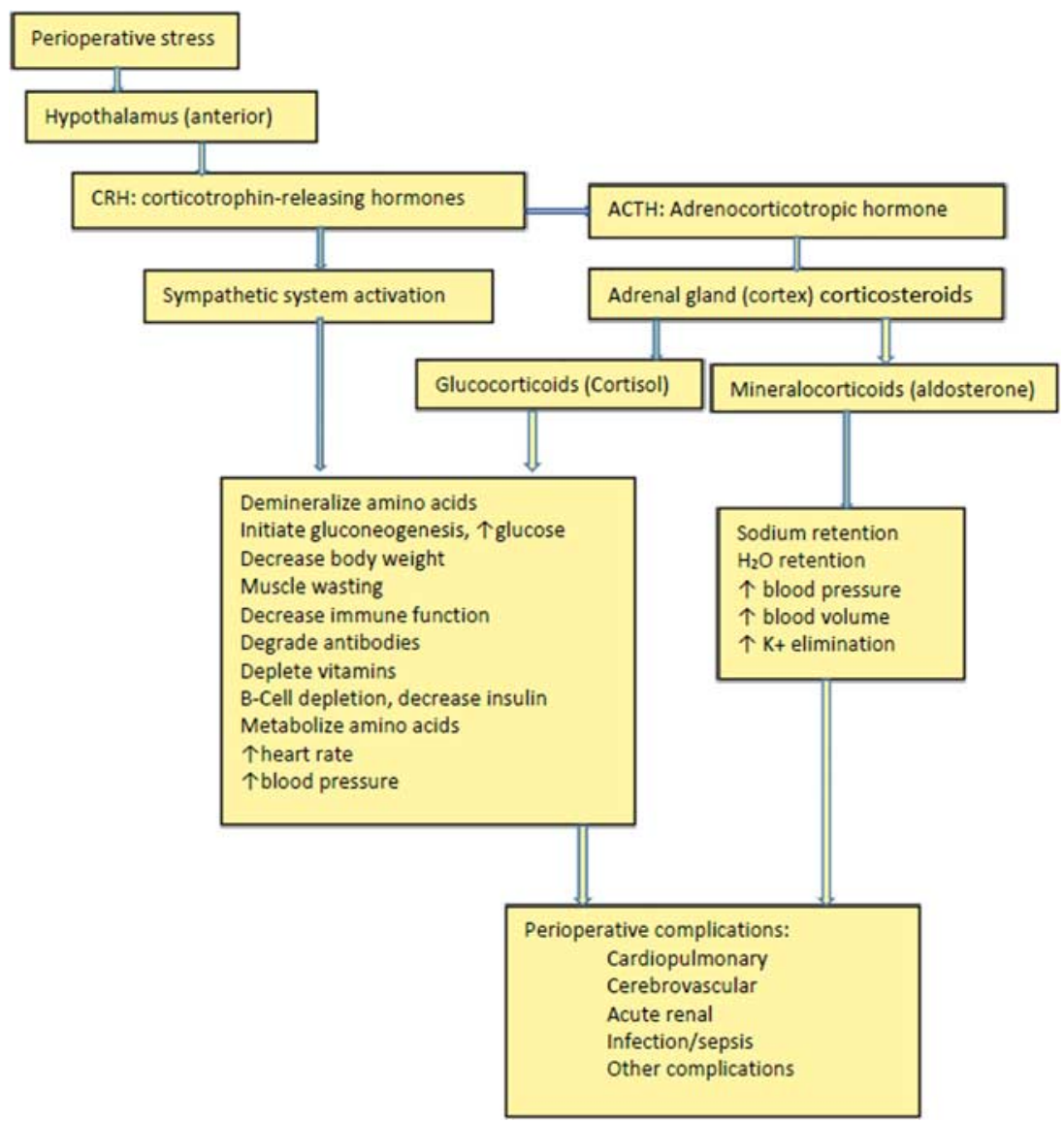

Figure 2. Perioperative stress induces pathophysiological changes. ${ }^{8} \frac{\text { full color }}{\frac{n}{\mathrm{n} l \mathrm{ine}} \mathrm{e}}$

4, and trigger the respective response. ${ }^{8,10}$ Inflammatory cytokines are formed and released in surgical patients. These cytokines include tumor necrotizing factor, multiple interleukins, etc. Cardiac surgery leads to an increased level of inflammatory cytokines, and statins may have some beneficial effects; rosuvastatin was found to attenuate these cytokine alterations. ${ }^{11}$

Postoperative patients admitted to ICU are at increased risk of developing gastrointestinal ischemia and potential bacterial translocation, which may cause bacteremia, endotoxemia, and even sepsis, multiple organ failure, and ultimately death. The duration of cardiopulmonary bypass $(\mathrm{CPB})$ is associated with increased intestinal permeability and increased risk of bacterial translocation. ${ }^{12}$

Perioperative stress can induce an upregulation of inducible nitric oxide synthase in organs such as the liver, lung, and spleen, and in the www.anesthesiaclinics.com

Copyright (C) 2017 Wolters Kluwer Health, Inc. All rights reserved. This paper can be cited using the date of access and the unique DOI number which can be found in the footnotes 
vascular system. ${ }^{13,14}$ Statins were found to have cardioprotective and anti-inflammatory properties perioperatively, likely by attenuation of the systemic inflammatory response and inhibition of nitric oxide metabolism. In this regard, pravastatin preloading has been demonstrated to significantly and dose relatedly result in an intense reduction in systemic inflammatory response with significant decrease in nitric oxide release and inducible nitric oxide synthase expression ${ }^{13}$ (Fig. 2).

The multiple systemic effects of perioperative stress lead to potentially harmful consequences, as shown in Table 1 .

\section{- Preoperative Patient Evaluation and Preparation}

\section{Preoperative Patient Preparations}

All elective patients should be evaluated preoperatively. ${ }^{15}$ The preanesthetic evaluation should include cardiopulmonary function, liver and renal function, metabolic status, and other potential comorbidities. Surgical risks should be stratified by using the standard ACS Risk Calculator. If risk of major adverse cardiac event exceeds $1 \%$, the patient should be referred for further cardiac testing/clearance. ${ }^{16}$ Methicillinresistant Staphylococcus aureus should also be screened. ${ }^{17}$ The patient's physical condition needs to be optimized preoperatively as well, including preoperative nutritional optimization, which must comprise the following: (1) daily fruit and vegetables; (2) starchy foods (rice, bread, pasta, and potatoes) at each mealtime; (3) protein-rich foods such as lean meat, fish, eggs, beans, etc.; and (4) limited intake of foods high in fat, sugar, and salt. ${ }^{18,19}$ The patient's family should be equally well informed as to what is expected for the whole perioperative course and how they should cooperate with health care providers to facilitate postoperative recovery.

Other preoperative preparation includes:

- Incentive spirometry training: this will help the patient become familiar with the use of incentive spirometry. Patients will do better if incentive spirometry is needed to prevent or to manage postoperative pneumonia. ${ }^{20}$

- Use of antiseptic soap before surgery.

Table 1. Neurohumoral Alterations as Responses to Perioperative Stress

Hypoxemia

$\uparrow \uparrow$ Myocardial oxygen demand $\rightarrow$ myocardial ischemia

Vasoconstriction may hinder wound healing and vascular anastomosis

Stress-induced exhaustion of energy and loss of muscle mass lead to weakness

(DVT, pneumonia)

$\uparrow \uparrow$ Risk of infection

Hypercoagulatory status $\rightarrow$ thromboembolic event

Sodium and water retention 
- Oral administration of statins.

- Preoperative physical preparedness.

- Patients should exercise for at least 15 minutes daily 2 weeks preoperatively.

- Patients should practice getting in and out of bed, standing up, and sitting down in a chair, and general everyday living activities within the home. ${ }^{18,19}$

\section{Preoperative Management on the Day of Surgery}

Avoidance of long fasting has been documented to be beneficial to surgical patients. ${ }^{18}$ Most ERAS ${ }^{\circledR}$ protocols suggest clear liquids up to 2 hours before surgery. ${ }^{15}$ Most protocols suggest a carbohydratecontaining drink 1 hour before arrival at the hospital. ${ }^{15}$ Surgical stress in the presence of fasting worsens the catabolic state, which may cause insulin resistance and delay recovery. Studies have shown that preoperative administration of oral carbohydrate liquid is safe and may have a favorable influence on perioperative variables of clinical outcome. $^{21}$

The patient should have a dose of anxiolytics to minimize anxiety and decrease preoperative stress. However, benzodiazepines should be avoided and opioids minimized for patients who are over 70 years of age. ${ }^{4}$

A study of 240 high-risk cardiac surgery patients with Cleveland Clinic Foundation score 6 or higher found that remote ischemic preconditioning was associated with significant absolute risk reduction, reduced incidence of 3-month composite endpoint major adverse kidney events and enhanced renal recovery in patients with acute kidney injury. ${ }^{22}$

\section{- Intraoperative Management}

\section{Prophylaxis of Infection}

Antibiotic(s) with appropriate bacterial coverage should be administered 30 minutes before surgical incision $(2 \mathrm{~h}$ for vancomycin and fluoroquinolones).$^{23}$ Some hospitals have strictly followed Surgical Care Improvement Project guidelines and have demonstrated decreased surgical-site infections by roughly 25\% from 2006 to 2010. Antibiotics may be given orally or IV, depending on the procedure.,24 $\mathrm{It}$ is abundantly clear that antibiotics should be given before central venous catheterization, which is typically needed for cardiovascular surgical procedures.

\section{Anxiolytic and Amnestic Agents}

The current trend is to minimize the use of benzodiazepines and to avoid benzodiazepines for patients who are over 70 years of age. ${ }^{4}$ 
However, benzodiazepines do provide an amnestic effect, which is important in cardiac surgical procedures, especially when a patient is hypotensive, necessitating reduction in anesthetic delivery.

\section{Opioid Use and Sympatholytic Therapy}

Opioid use in cardiac surgery has been steadily declining over the last 2 decades; however, opioids block perioperative stress response. Epidural and intrathecal administration of opioids have been shown to help attenuate perioperative stress responses. ${ }^{25}$

\section{Standardized Preemptive Multimodal Analgesia}

The multimodal approach should include $\geq 2$ of the following categories of medications: opioids, nonsteroidal anti-inflammatory drugs, acetaminophen, and gabapentin. ${ }^{15}$ Analgesic agents are to be given preoperatively and in the early stages during surgical procedures. Smaller doses should be considered to avoid over-sedated patients after surgery.

If applicable, thoracic epidural analgesia should be considered for cardiac surgery, although there is an increased risk of complications due to systemic anticoagulation. Sen and colleagues prospectively studied 60 patients scheduled for coronary artery bypass grafts (CABG) surgery. They discovered thoracic epidural analgesia with fentanyl and bupivacaine provided optimal postoperative analgesia at rest and during coughing in patients following coronary bypass grafting surgery. ${ }^{25}$

Negro Neto and colleagues studied the intrathecal administration of sufentanil for coronary artery bypass grafts $(\mathrm{CABG})$ patients, measuring patients' hemodynamic variables, interleukin-6, blood levels of cardiac troponin I, tumor necrosis factor, and B-type natriuretic peptide during and after surgery. The results demonstrated that the sufentanil group required remarkably less dopamine support than the control group and less increases in remifentanil doses. ${ }^{26}$

\section{Anti-inflammatory Therapy}

Systemic inflammation has been well recognized in cardiac surgery. Such inflammation is induced by tissue injury, contact with the artificial surface of the bypass circuit, hypothermia and reduction of pulmonary blood flow during surgery, as well as perioperative stress. Steroids are recommended by some cardiac surgery programs, although there is no consensus on whether steroids should be routinely administered to all cardiac surgery patients. ${ }^{27}$ High spinal anesthesia added to general anesthesia can potentially attenuate systemic inflammatory responses. ${ }^{28}$ 


\section{Fluid Management and Blood Transfusion}

Cardiac patients usually have invasive monitors easily allowing for monitoring of volume status. Central venous pressure, stroke volume variations, and transesophageal echocardiography can be used to directly estimate volume status. Avoidance of fluid overload is advised, especially in patients undergoing $\mathrm{CPB}$, as the $\mathrm{CPB}$ circuit has about $2 \mathrm{~L}$ of priming fluid. Patients undergoing cardiac surgery are more likely to receive blood transfusion because of the high risk of blood loss. The blood transfusion guidelines are not universally accepted, and a great variability exists in the use of blood products between countries, institutions, and practitioners. The hemoglobin level to initiate transfusion has been steadily declining over the last decades. ${ }^{29}$ Transfusionassociated acute lung injury has been increasingly recognized as a complication of transfusion. Hospitals need to develop protocols to avoid or minimize blood transfusion, which can include reversal of anticoagulant, acute normovolemic hemodilution, antifibrinolytic agents, and other hemostatic pharmacological agents. ${ }^{29,30}$

\section{Vasoactive Medications}

There are tremendous variations in selecting vasoactive medications intraoperatively at different institutions. Discrepancies exist in several areas. Some physicians use epinephrine and dobutamine as a combination to treat patients with low cardiac output and hypotension. There are studies demonstrating that relatively weak sympathomimetic agents (dobutamine) can potentially antagonize potent sympathomimetic agents (epinephrine). If synergistic inotropic and/or vasoconstricting effects are needed, they may not be a favorable combination. ${ }^{31}$ Some surgeons request nitroglycerin for spasm prevention of the radial or mammary artery conduit graft, whereas others use nicardipine. We believe nicardipine is a better choice. Nitroglycerin is known as a coronary artery dilator; however, radial and mammary artery grafts are anatomically and physiologically arterial vessels. They likely would respond to a direct arterial dilator (eg, nicardipine) better than the venous dilator nitroglycerin. ${ }^{32,33}$

Long-acting $\beta$-adrenergic receptor antagonists (atenolol, labetalol) are often administered during cardiac surgical procedures. $\beta 1$-adrenergic receptors are critical in maintaining cardiac rhythm and myocardial contractility; if blocked by selective $\beta 1$-adrenergic receptor antagonists, patients may develop bradycardia and/or hypotension, and sympathomimetic agents such as epinephrine, norepinephrine, or dobutamine may not be able to activate/stimulate $\beta 1$-adrenergic receptors. We suggest only using selective short-acting $\beta 1$-adrenergic receptor antagonists intraoperatively (eg, esmolol). Catecholamine administration has been 
associated with adverse clinical outcomes; hence, epinephrine should be cautiously used perioperatively. ${ }^{34-36}$

\section{Blood Glucose Level}

High blood glucose levels have been associated with elevated morbidity and mortality in surgical patients. ${ }^{37}$ Many studies with regard to hyperglycemia in cardiac surgery have been published; however, those studies have used wide variations in the definitions of hyperglycemia, methodology, timing, and dosing of insulin administration. More importantly, hypoglycemia can cause significant problems. After the Leuven study described better outcomes with tighter glucose parameters, ${ }^{37}$ tight glycemic control became popular. However, several large-scale multicenter studies have led practice guidelines to be modified recently, favoring conventional, less-tight control. It appears beneficial that diabetic patients can be managed with blood glucose allowed up to $180 \mathrm{mg} / \mathrm{dL}$ and nondiabetic patients at $<140 \mathrm{mg} / \mathrm{dL}$. ${ }^{37}$

\section{Invasive Monitors}

The overall trend is a decrease in invasive monitoring and increased use of less-invasive or noninvasive monitoring. Transesophageal echocardiography is increasingly used as an intraoperative monitor in cardiac surgery and can provide almost all parameters that a Swan-Ganz catheter can provide, except mixed venous oxygen saturation. Less complicated cardiac surgery patients only need a central venous catheter, ${ }^{38}$ which is unlikely to be replaced by other volume monitors, because it functions as a large-bore intravenous access as well.

\section{Intraoperative Normothermia}

Surgical patients are prone to lose heat intraoperatively. General anesthesia changes the regulatory set-point in the brain for defense against cold exposure, rendering the patient poikilothermic. Hypothermia will pose significant stress to surgical patients. Therefore, temperature in the operative rooms should be maintained between $20^{\circ}$ $\mathrm{C}$ and $25^{\circ} \mathrm{C}$, and many other strategies will be utilized to keep surgical patients normothermic. $^{2}$

\section{Surgical Hemostasis}

Thorough surgical hemostasis intraoperatively is critical to the success of the surgery and the recovery of the patient. Many "bring back" surgical cases are due to rebleeding, which causes significant postoperative morbidity and mortality. 


\section{Minimally Invasive Approaches}

Minimally invasive techniques have been well documented to facilitate postoperative recovery in many surgical subspecialties. ${ }^{39}$ Robotic techniques allow surgeons to perform cardiac surgery endoscopically rather than through sternotomy. This approach facilitates multiple cardiac procedures involving the mitral valve, atrial septal defects, and coronary artery system. In Europe, robotic coronary surgery has been performed safely and effectively in the last 15 years with very few perioperative complications. Robotic mitral valve surgery has also been increasingly utilized in the last 5 to 6 years. ${ }^{40}$ Integration of robotic endoscopic cardiac surgery into ERAS is not only feasible and safe but also potentially synergistic with respect to postoperative recovery. One retrospective study showed significantly lower blood transfusion, shorter ICU duration by 24 hours, and a 4-day decrease in hospital stay in the robotic endoscopic CABG group. ${ }^{39}$

\section{- Postoperative Management}

\section{Ventilation Management}

With currently available techniques, the majority of cardiac surgical patients will need postoperative mechanical ventilation. Prolonged intubation may cause ventilator-associated pneumonia (VAP) as a complication. The incidence of VAP after cardiac surgery is estimated to be as high as $30 \%$, and S. aureus and Pseudomonas aeruginosa are the most commonly involved microorganisms. ${ }^{41}$ Prevention of VAP may need to start with a protective ventilation mode intraoperatively. ${ }^{42}$ Ventilatory protective settings include low tidal volume, and potentially positive end-expiratory pressure and recruitment maneuvers. Postoperatively, low tidal volume and tight control of driving pressure should be applied. Ventilation driving pressure should be maintained as low as possible. ${ }^{26}$ Patients should be extubated as soon as they reach extubation criteria and have no other major medical issues. ${ }^{43}$ Physiotherapy, early mobilization, and respiratory monitoring may play an important role in preventing postoperative pulmonary complications and enhance recovery. ${ }^{44}$

\section{Postoperative Sedation}

As most cardiac surgery patients will need mechanical ventilation postoperatively in the ICU, sedation of these patients will be paramount. Postoperative sedation for patients after cardiac surgery in the ICU needs to be tailored to facilitate the patient's anticipated emergence, to reduce postoperative discomfort and anxiety, and to accommodate frequent suctioning and physiotherapy. Benzodiazepines and propofol 
are commonly utilized in the ICU to sedate ventilated patients, with dexmedetomidine being increasingly used as well. ${ }^{45}$

\section{Pain Management}

Multimodal analgesia is highly recommended by many expert consensus statements and guidelines. ${ }^{15}$ Inadequate control of postoperative pain may lead to prolonged immobilization, insufficient lung expansion and respiratory dysfunction, and patient's inability to cough related to median sternotomy. Hence, the duration of mechanical ventilation, length of ICU stay, and length of hospital stay will also be significantly increased. Thus, adequate analgesia after cardiac surgery is critically important in the recovery of patients from cardiac surgery. ${ }^{46}$

Opioids are the choice of potent analgesic agents. However long-acting opioids should be minimized in postoperative analgesia unless the patient is expected to be mechanically ventilated for an extended period. Nonsteroidal anti-inflammary drugs are an excellent adjunct for postoperative analgesia, but their potential renal, gastrointestinal, and coagulative complications and their analgesic potency are barriers for their sole or routine use in cardiac surgical patients. ${ }^{47}$

The major source of pain in cardiac surgery patients is from the median sternotomy and the mediastinal drainage tube sites. The sternum is innervated by the anterior and posterior branches of intercostal nerves, which can be blocked by parasternal local anesthetic infiltration. This block is simple and easy to perform and is not contraindicated even in anticoagulated patients. ${ }^{48}$ Neuraxial block has also been recommended ${ }^{4}$ as a sole anesthetic for noncardiac surgical procedures or as an adjunct analgesic measure for noncardiac and cardiac surgical procedures.

\section{Early Mobilization Postoperatively With Physiotherapy Assistance}

Immobilized critically ill patients are at risk to develop pneumonia, neuromyopathy, sepsis, and multiorgan failure. ${ }^{49}$ Early mobility interventions can have enormous benefits: improved range of motion, decreased incidence of pneumonia, better muscle strength and functional status, and decreased length of ICU and hospital stay. Early mobilization is also possible in mechanically ventilated patients. ${ }^{49}$ Most ERAS protocols recommend early mobilization. ${ }^{15,50}$

\section{Facilitation of Gastrointestinal Function Recovery}

Gastrointestinal function recovery can be enhanced by adopting early oral nutrition, chewing gum use, and pharmacologically promoting gastrointestinal motility. Alvimopam, a selective $\mu$-opioid receptor blocker, can be administered after starting oral intake until return of 
bowel function. Early oral nutrition can promote intestinal peristalsis, and improve nutrition and fluid intake, thus decreasing intravenous fluid requirement and potentially decrease bacterial translocation, thus lowering the risk of endotoxemia and sepsis. ${ }^{15}$

\section{Early Removal of Nasogastric Tube, Foley Catheter, and Drainage Tube(s)}

All indwelling catheters and tubes will potentially increase the incidence of infection and sepsis. Hence, most ERAS protocols and expert consensus recommend minimizing the use of indwelling catheters and tubes. If medically needed, those inserted catheters and tubes should be removed as soon as medical conditions allow. ${ }^{15}$ For patients needing indwelling central venous catheterization, the catheter should be removed and replaced in no $>1$ week, or whenever the patient's body temperature escalates.

\section{Fluid Management}

Fluid management based on ERAS protocol should be viewed as a continuum throughout the preoperative, intraoperative, and postoperative phases. Any phase with suboptimal care can potentially compromise the overall results. Excessive salt should generally be avoided because these patients usually do not have the same capability to excrete sodium and chloride postoperatively. The aim should be to provide low-sodium and low-volume fluids to postoperative patients. Goal-targeted therapy should be utilized if available, especially in high-risk patients. ${ }^{22,51}$ After oral fluid intake starts, intravenous fluid administration should be reduced or discontinued. An average patient without existing fluid deficits or ongoing losses should be encouraged to orally intake $1.75 \mathrm{~L} / \mathrm{d}$. Without other medical concerns or in the state of normovolemia, permissive oliguria should be tolerated. ${ }^{51}$

\section{- ERAS $^{\circledR}$ Variables in Cardiac Surgery ${ }^{4,52}$}

The following variables are useful metrics for the enhanced recovery pathway to keep track of clinical outcomes, measure compliance with the protocol, and to conduct research:

- Incidence and severity of postoperative complications

- Time to extubation in the ICU

- Time to oral intake and recovery of gastrointestinal function

- Time to early mobilization

- Incidence of surgical complications

- ICU stay

- Total hospital stay

- Patient satisfaction

- Total hospital costs

www.anesthesiaclinics.com 


\section{- Current ERAS Studies in Cardiac Surgery and Outcomes}

A widely accepted ERAS protocol for cardiac surgery will take some efforts. Perkins and colleagues reported implementing the ERAS program in England beginning in 2009. Their patients have been successfully admitted for cardiac surgery on the day of surgery. Their ERAS program is supported by multidisciplinary preadmission clinics 1 week preoperatively for all cardiothoracic patients and adopted for all first-time CABG patients. They started ERAS with gastrointestinal surgery in 2009 and cardiac surgery in $2013 .{ }^{53}$ Cardiac surgery without $\mathrm{CPB}$ can also be included in the ERAS cardiac surgery program. Fleming and colleagues adopted a protocol including preoperatively detailed patient evaluation, avoiding prolonged preoperative fasting, preoperative carbohydrate intake, multimodal analgesia avoiding long-acting opioids, prophylaxis of postoperative nausea and vomiting, early enteral nutrition, and early mobilization. They found that ERAS produced significantly lower postoperative complications (acute kidney injury, atrial fibrillation, infections, respiratory failure, acute myocardial infarction, and death). They believe that ERAS in cardiac surgery is feasible and may improve postoperative morbidity and mortality. ${ }^{6}$

Kenny and colleagues also reported favorable results in relatively elderly patients who underwent cardiac surgery, with the mean age being 67.5 in the CABG group and 72.9 in the aortic valve replacement group. Their ICU stay ranged from 0.7 to 5.1 days (median, $0.9 \mathrm{~d}$ ) for the CABG group and 0.7 to 18.8 days (median, $0.9 \mathrm{~d}$ ) for the aortic valve replacement group. Hospital stay ranged from 3 to 8 days (median, $5 \mathrm{~d}$ ) for CABG and 5 to 35 days (median, $9 \mathrm{~d}$ ) for aortic valve replacement. These results represented a $17 \%$ reduction in hospital stay for CABG patients. ${ }^{54}$ As multimodal perioperative pain therapy significantly contributed to patient care, ${ }^{55}$ ERAS has the potential to contribute to perioperative care in cardiac surgery.

\section{- Summary}

Cardiac ERAS represents a common sense appreciation for the mediation or modulation of the stress response to surgery, anesthetic management to facilitate recovery, and a heavy emphasis on practical strategies initiated before surgery to enhance recovery postoperatively. This review builds upon ERAS investigations over the past 20 years and represents the best practice strategies for cardiac surgical patients. These strategies require a buy-in from all stakeholders, including surgeons, nurses, and administration. Forming a Cardiac ERAS group at each hospital facility will help build team consensus and gain support for its 
implementation, with anesthesia providers playing a critical role in this process. At present, reimbursement is typically bundled, and this means that improved recovery and ultimate discharge home without return to the hospital is financially beneficial to the facility and directly improves outcomes of patients.

The authors declare that they have nothing to disclose.

\section{- References}

1. Kehlet H. Multimodal approach to control postoperative pathophysiology and rehabilitation. Br J Anaesth. 1997;78:606-617.

2. Kehlet H, Wilmore DW. Multimodal strategies to improve surgical outcome. Am J Surg. 2002;183:630-641.

3. Zakhary W, Lindner J, Sgouropoulou S, et al. Independent risk factors for fast-track failure using a predefined fast-track protocol in preselected cardiac surgery patients. J Cardiothorac Vasc Anesth. 2016;29:1461-1465. 2015.

4. Miller TE, Thacker JK, White WD, et al. Enhanced Recovery Study Group. Reduced length of hospital stay in colorectal surgery after implementation of an enhanced recovery protocol. Anesth Analg. 2014;118:1052-1061.

5. Abeles A, Kwasnicki RM, Darzi A. Enhanced recovery after surgery: current research insights and future direction. World J Gastrointest Surg. 2017;9:37-45.

6. Fleming IO, Garratt C, Guha R, et al. Aggregation of marginal gains in cardiac surgery: feasibility of a perioperative care bundle for enhanced recovery in cardiac surgical patients. J Cardiothorac Vasc Anesth. 2016;30:665-670.

7. Hardman G, Bose A, Saunders H, et al. Enhanced recovery in cardiac surgery. World Society of Cardiothoracic Surgeons 25th Anniversary Congress, Edinburgh, UK, September 19-22, 2015.

8. Liu H, Yan H, Christian S, et al. Physiological derailments in trauma patients. In: Scher Corey S, ed. Anesthesia for Trauma: New Evidence and New Challenges (ISBN: 978-14939-0908-7). Berlin: Springer; 2005:45-66.

9. Chaudry IH, Ayala A. Mechanism of increased susceptibility to infection following hemorrhage. Am J Surg. 1993;165 (2A suppl):59SY67S.

10. Mollen KP, Anand RJ, Tsung A, et al. Emerging paradigm: toll-like receptor 4 sentinel for the detection of tissue damage. Shock. 2006;26:430Y437.

11. Sánchez-Aguilar M, Tapia-Pérez JH, Sánchez-Rodríguez JJ, et al. Effect of rosuvastatin on cytokines after traumatic head injury. J Neurosurg. 2013;118:669-675.

12. Adamik B, Kübler A, Gozdzik A, et al. Prolonged cardiopulmonary bypass is a risk factor for intestinal ischaemic damage and endotoxaemia. Heart Lung Circ. 2016;26: 717-723.

13. Castaño M, González-Santos JM, López J, et al. Effect of preoperative oral pravastatin reload in systemic inflammatory response and myocardial damage after coronary artery bypass grafting. A pilot double-blind placebo-controlled study. J Cardiovasc Surg (Torino). 2015;56:617-629.

14. Thiemermann C, Szabo C, Mitchell JA, et al. Vascular hyporeactivity to vasoconstrictor agents and hemodynamic decompensation in hemorrhagic shock is mediated by nitric oxide. Proc Natl Acad Sci U S A. 1993;90:267Y271.

15. http://aserhq.org/protocols.

16. Hiratzka LF, Creager MA, Isselbacher EM, et al. Surgery for aortic dilatation in patients with bicuspid aortic valves: a statement of clarification from the American 
College of Cardiology/American Heart Association Task Force on Clinical Practice Guidelines. J Am Coll Cardiol. 2016;67:724-731.

17. www.uhs.nhs.uk/Media/Controlleddocuments/Patientinformation/Heartandlungs/ Cardiac-surgery-enhanced-recovery-patient-information.pdf.

18. Kehlet H, Wilmore DW. Surgical care-how can new evidence be applied to clinical practice? Colorectal Dis. 2010;12:2-4.

19. www.papworthhospital.nhs.uk/docs/leaflets/PI-82-Enhancing-your-recovery-aftercardiac-surgery.pdf.

20. Holubar SD, Hedrick T, Gupta R, et al. Perioperative Quality Initiative (POQI) I Workgroup. American Society for Enhanced Recovery (ASER) and Perioperative Quality Initiative (POQI) joint consensus statement on prevention of postoperative infection within an enhanced recovery pathway for elective colorectal surgery. Perioper Med (Lond). 2017;6:4.

21. Bilku DK, Dennison AR, Hall TC, et al. Role of preoperative carbohydrate loading: a systematic review. Ann R Coll Surg Engl. 2014;96:15-22.

22. Zarbock A, Kellum JA, Van Aken H, et al. Long-term effects of remote ischemic preconditioning on kidney function in high-risk cardiac surgery patients: follow-up results from the renal RIP trial. Anesthesiology. 2017;126:787-798.

23. Engelman R, Shahian D, Shemin R, et al. Workforce on Evidence-Based Medicine, Society of Thoracic Surgeons. The Society of Thoracic Surgeons practice guideline series: antibiotic prophylaxis in cardiac surgery, part II: antibiotic choice. Ann Thorac Surg. 2007;83:1569-1576.

24. www.merckmanuals.com/professional/special-subjects/care-of-the-surgical-patient/ antibiotic-prophylaxis-for-surgical-procedures.

25. Sen AC, Rajan S, Balachandran R, et al. Comparison of perioperative thoracic epidural fentanyl with bupivacaine and intravenous fentanyl for analgesia in patients undergoing coronary artery bypass grafting surgery. Anesth Essays Res. 2017;11:105-109.

26. Neto AS, Hemmes SN, Barbas CS, et al. Association between driving pressure and development of postoperative pulmonary complications in patients undergoing mechanical ventilation for general anaesthesia: a meta-analysis of individual patient data. Lancet Respir Med. 2016;4:272-280.

27. Liu H, Tariq R, Liu GL, et al. Pharmacological cerebral protection in cardiac surgery: an update. J Anesth Perioper Med. 2017;4:23-37.

28. Lee TW, Kowalski S, Falk K, et al. High spinal anesthesia enhances anti-inflammatory responses in patients undergoing coronary artery bypass graft surgery and aortic valve replacement: randomized pilot study. PLoS One. 2016;11:e0149942.

29. Brouwers C, Hooftman B, Vonk S, et al. Benchmarking the use of blood products in cardiac surgery to stimulate awareness of transfusion behaviour: results from a fouryear longitudinal study. Neth Heart J. 2017;25:207-214.

30. ASA. http://www.asahq.org/quality-and-practice-management/standards-and-guidelines. Accessed October 15, 2014.

31. Prielipp RC, MacGregor DA, Royster RL, et al. Dobutamine antagonizes epinephrine's biochemical and cardiotonic effects: results of an in vitro model using human lymphocytes and a clinical study in patients recovering from cardiac surgery. Anesthesiology. 1998;89: $49-57$.

32. Liu H, Kalarickal PL, Yan H, et al. Pharmacological management of perioperative pulmonary hypertension: an update. J Anesth Perioper Med. 2015;2:316-324.

33. Liu H, Fox CJ, Zhang S, et al. Cardiovascular pharmacology: an update. Anesthesiol Clin. 2010;28:723-738.

34. Mebazaa A, Parissis J, Porcher R, et al. Short-term survival by treatment among patients hospitalized with acute heart failure: the global ALARM-HF registry using propensity scoring methods. Intensive Care Med. 2011;37:290-301. 
35. Liu H, Sangkum L, Liu G, et al. Effects of epinephrine on angiogenesis-related gene expressions in cultured rat cardiomyocytes. J Biomed Res. 2016;30:380-385.

36. Callaway CW. Questioning the use of epinephrine to treat cardiac arrest. JAMA. 2012;307:1198-1200.

37. van den Berghe $\mathrm{G}$, Wouters $\mathrm{P}$, Weekers $\mathrm{F}$, et al. Intensive insulin therapy in critically ill patients. N Engl J Med. 2001;345:1359-1367.

38. Sangkum L, Liu GL, Yu L, et al. Minimally invasive or noninvasive cardiac output measurement: an update. J Anesth. 2016;30:461-480.

39. Zaouter C, Imbault J, Labrousse L, et al. Association of robotic totally endoscopic coronary artery bypass graft surgery associated with a preliminary cardiac enhanced recovery after surgery program: a retrospective analysis. J Cardiothorac Vasc Anesth. 2015;29:1489-1497.

40. Pettinari M, Navarra E, Noirhomme P, et al. The state of robotic cardiac surgery in Europe. Ann Cardiothorac Surg. 2017;6:1-8.

41. He S, Chen B, Li W, et al. Ventilator-associated pneumonia after cardiac surgery: a meta-analysis and systematic review. J Thorac Cardiovasc Surg. 2014;148:3148-3155.

42. Serpa Neto A, Schultz MJ, Gama de Abreu M. Intraoperative ventilation strategies to prevent postoperative pulmonary complications: systematic review, meta-analysis, and trial sequential analysis. Best Pract Res Clin Anaesthesiol. 2015;29:331-340.

43. Higgins TL. Pro: early endotracheal extubation is preferable to late extubation in patients following coronary artery surgery. J Cardiothorac Vasc Anesth. 1992;6:488-493.

44. Ball L, Costantino F, Pelosi P. Postoperative complications of patients undergoing cardiac surgery. Curr Opin Crit Care. 2016;22:386-392.

45. Constantin JM, Momon A, Mantz J, et al. Efficacy and safety of sedation with dexmedetomidine in critical care patients: a meta-analysis of randomized controlled trials. Anaesth Crit Care Pain Med. 2016;35:7-15.

46. Ozturk NK, Baki ED, Kavakli AS, et al. Comparison of transcutaneous electrical nerve stimulation and parasternal block for postoperative pain management after cardiac surgery. Pain Res Manag. 2016;2016:4261949.

47. Liu H, Fox CJ, Kaye AD. Pharmacology for cardiac surgery. In: Kaye AD, Kaye AM, Urman RD, eds. Essentials of Pharmacology for Anesthesia, Pain Medicine, and Critical Care. Berling, Germany: Springer; 2015:609-643.

48. McDonald SB, Jacobsohn E, Kopacz DJ, et al. Parasternal block and local anesthetic infiltration with levobupivacaine after cardiac surgery with desflurane: the effect on postoperative pain, pulmonary function, and tracheal extubation times. Anesthes Analg. 2005;100:25-32.

49. Hruska P. Early mobilization of mechanically ventilated patients. Crit Care Nurs Clin North Am. 2016;28:413-424.

50. Giménez-Milà M, Klein AA, Martinez G. Design and implementation of an enhanced recovery program in thoracic surgery. J Thorac Dis. 2016;8(Suppl 1):S37-S45.

51. Miller TE, Roche AM, Mythen M. Fluid management and goal-directed therapy as an adjunct to Enhanced Recovery After Surgery (ERAS). Can J Anaesth. 2015;62:158-168.

52. Mathias JM ERAS improves outcomes, shortens length of stay. OR Manager. March 17,2016

53. Perkins A, McAdam P, H Saunders H, et al. Available at: www.actacc.org/abstracts/ abstractprint.asp?a=SIJEGOKI243478SAOCOM25062014_91. [Abstract].

54. Kenny L, Pillay T, Kinnersley D. The enhanced recovery programme pilot: can we achieve better outcomes and shorter stays for cardiac surgical patients? J Cardiothorac Surg. 2013;8(Suppl 1):O1.

55. Gritsenko K, Khelemsky Y, Kaye AD, et al. Multimodal therapy in perioperative analgesia. Best Pract Res Clin Anaesthesiol. 2014;28:59-79. 